\title{
Investigation of a Two-Qubit Decoherence by Using Bloch Vectors
}

\author{
F. AHMADI $^{a, *}$ AND S. JAMI ${ }^{b}$ \\ ${ }^{a}$ Ph.D. Student, Faculty of Science, Dept. of Physics, Universiti Teknologi Malaysia, \\ 81310 UTM Johor Bahru, Johor, Malaysia \\ ${ }^{b}$ Dept. of Physics, Islamic Azad University, Mashhad Branch, Mashhad, Iran
}

(Received July 14, 2013)

\begin{abstract}
The loss of the coherence happens due to the interaction between the desired system and its surroundings. Addressing decoherence is one of the main concepts in the study of quantum channels to access their potential for various scale. In this process the information of the system is penetrated into the environment. Therefore, the measurement of the environment could be used as the error correction method. Phase damping channels principally belong to random unitary channels and can be corrected by classical information. This paper presents a method to generate non-random unitary phase damping channels based on the Bloch vectors in two qubits systems. A phase damping channel which consisted of a two qubits system and a single qubit environment was investigated. The results demonstrated that the phase damping channels belong to random unitary dynamics if the three-dimensional tetrahedron volume spanned by the Bloch vectors in $\mathbb{R}^{3}$ is not zero, or, the same hyperplane in $\mathbb{R}^{3}$ was not pointed by the Bloch vectors. It is found that the Bell state belongs to random unitary class and can be corrected based on classical information obtained from environmental measurements.
\end{abstract}

DOI: 10.12693/APhysPolA.125.1088

PACS: 03.65.Yz, 03.67.Pp, 03.65.-w

\section{Introduction}

Closed system is an ideal system that does not interact with the outside world, whereas real system has undesirable interaction with the environment $[1,2]$. The interaction between an open quantum system and its surroundings causes correlation between the states of the system and of the environment that is called decoherence [3]. Decoherence arises as a result of coupling of the desired system and quantum environment. Decoherence is the most important obstacle to use quantum states in larger and larger scale [4]. Phase damping channel is one type of decoherence in which non-diagonal elements of density matrix are altered due to the coupling of the system and environment; however, diagonal elements of density matrix remain unchanged. Two sources are responsible for decoherence. The most important source is correlation between system and its surrounding that is a direct outcome of an open quantum system. Classical fluctuations of fields are the second source of decoherence [5]. Decoherence is usually explained without quantum environment that these channels are denoted as random unitary (RU) channels. These channels can be corrected by classical information of environment [6-9] and are more important in practical view. For instance, decoherence can be regulated by RU dynamics in nuclear magnetic resonance and quantum computers which are based on trapped ions $[2,4]$.

For single qubits and qutrits, error correction method rests on measurement of the quantum environment in any

*corresponding author; e-mail: ahmadi.fahimeh25@yahoo.com phase damping channels, therefore these channels always belong to RU maps $[9,10]$. In contrast, there are some cases where correcting decoherence by measuring the environment is impossible and it might be a problematic subject when environment is too big. Furthermore, in some cases where the whole environment is accessible, correcting decoherence is unattainable.

Landau and Streater explained that in the Hilbert spaces of dimension $N>3$, there are some situations in which error correction method by measuring the environment is impossible [10]. Helm et al. developed a geometrical measurement by using the Bloch sphere picture to determine the non-RU channels $[7,8]$. In this paper a new method is presented using expansion of considerable state based on Hamiltonian's eigenvalues to investigate the non-RU dynamics.

\section{Quantum channels}

In order to describe dynamical evolution of an open quantum system, it is supposed that the system and its environment are separable and there is no correlation between them. $\varepsilon(\rho)$ is a mapping that describes the evolution of the system in which it acts on initial density operator. Then, density matrix is transformed into new density operator as follows [2]:

$$
\rho^{\prime}=\varepsilon(\rho) .
$$

Dynamic of quantum system is a completely positive map (or quantum channel) that is shown in term of the Kraus operators $\left(K_{i}\right)$ as follows:

$$
\rho_{\mathrm{s}}^{\prime} \rightarrow \varepsilon\left(\rho_{\mathrm{s}}\right)=\sum_{i} K_{i} \rho_{\mathrm{s}} K_{i}^{\dagger} .
$$

As it can be seen in the equation, there is a set of unitary operators instead of one unitary operator. Therefore 
some of the pure states of the system may be entangled to some of the environmental state. For $N$-dimension of the Hilbert space these completely positive maps are at most $N^{2}$ Kraus operators [8]. It is generally supposed that the channel is trace-preserving $\left(\sum_{i} K_{i}^{\dagger} K_{i}=\mathbb{1}\right)$. In addition, the channel is unital or doubly stochastic if the completely positive state is mapped unto itself $\left(\sum_{i} K_{i} K_{i}^{\dagger}=\mathbb{1}\right)$.

\subsection{Phase damping channel}

Decoherence of an open system can be caused by the increase of the entanglement between system and environment $[1,7,8]$. Pure state is turned into mixed state as the consequence of interaction between the system and environment $[2,3,7,11]$. In this process information of the system is penetrated into environment. In addition, non-diagonal elements of reduced density matrix are changed. Phase damping channels are the simplest map among other maps. Phase damping channels is defined in phase damping basis as follows:

$$
\{|n\rangle, 1 \leq n \leq N\},
$$

in which $N$ is the dimension of quantum system. The diagonal elements remain unchanged, $\langle n|\rho| n\rangle=$ const, with $n=1, \ldots, N$ in phase damping channel and the changing in non-diagonal elements is the only impact of environment $\langle n|\rho| m\rangle$ with $n \neq m[7,8]$. Hence, Kraus operators are diagonal in this basis $K_{i}=\operatorname{diag}\left(a_{i 1}, a_{i 2}, \ldots, a_{i N}\right)$, and consequently the whole map is diagonal

$$
\rho_{m n}^{\prime}=\rho_{m n}\left\langle a_{n} \mid a_{m}\right\rangle,
$$

in which $\left|a_{n}\right\rangle=\left(a_{1 n}, a_{2 n}, \ldots, a_{r n}\right)$ is a set of $N$ normalized complex vectors. It is concluded that the phase damping channels are the only diagonal doubly stochastic quantum channels. It was proven that there are some nonunitary extremal doubly stochastic completely positive maps which are not able to be described without quantum environment. In this case the system coupling with the environment $\left|a_{n}\right\rangle$ may be recognized as environmental quantum states.

\subsection{Random unitary channel}

Quantum channels are used for data transmission and decoherence happens in these channels because of inevitable interaction between system and environment. Therefore, there is a need to develop error correction techniques in order to use quantum states in huge quantum information tasks [2]. Using the information obtained from the environment is one of the most important error correction schemes. RU channels are corrected using classical information from environment. RU channels can be described as a convex combination of unitary transformation as follows [12-15]:

$$
\varepsilon(\rho)=\sum_{i} P_{i} U_{\mathrm{i}} \rho U_{i}^{\dagger}, \quad P_{i}>0, \quad \sum_{i} P_{i}=1,
$$

in which $\rho$ is density operator and $P_{i}$ are the scalars that form a probability distribution and $U_{i}$ are unitary operators. RU channel satisfies the following equation, so it is unital [13]:

$$
\varepsilon(\mathbb{1})=\mathbb{1} \text {. }
$$

If the only error mechanism is a classical uncertainty, it is possible to describe this map as the mixture of unitary maps. The mathematical description of this mixture is an RU map. On the other hand, if decoherence arises from coupling of the system and a quantum environment, this map can no longer be described by an RU map and this channel can be corrected by using classical information obtained from the environment as well [12].

A unital completely positive trace preserving map is RU map if its state representative is described as follows:

$$
\phi=\sum_{i} P_{i}\left(U_{i} \otimes \mathbb{1}\right)|I\rangle\langle I|\left(U_{i}^{\dagger} \otimes \mathbb{1}\right),
$$

in which $\phi$ is called state representative of completely positive map that it assigns to each completely positive map $H_{\text {in }}$ to $H_{\text {out }}$ a state on $H_{\text {in }} \otimes H_{\text {out }}$. In addition, $|I\rangle$ denotes maximally entangled (ME) state vector

$$
|I\rangle:=\frac{1}{\sqrt{N}} \sum_{i=1}^{N}|i, i\rangle,
$$

in which $\{|i\rangle\}$ are orthogonal basis in $H_{\text {in }}$. In addition, a pure state will be an RU map if this pure state is ME. Also, ME states consist of the Bell states. In other words, if a pure state is a convex combination of ME states, it will be RU dynamics. Evidently, the Bell state is a part of RU class [4].

\section{RU and phase damping channels for two qubits systems}

Landau and Streater demonstrated that, in the set of diagonal doubly stochastic quantum channels in the Hilbert spaces $N>3$, there are some non-unitary extremal maps that perfect improvement is not possible in spite of complete monitoring the environment [10]. A diagonal doubly stochastic channel $\varepsilon$ is considered as extremal channel if $\varepsilon$ admits an expression of form Kraus operators $\varepsilon(\rho)=\sum_{i=1}^{r} K_{\mathrm{i}} \rho K_{i}^{\dagger}$, where $\left.K_{i}^{\dagger} K_{j}\right\}_{i, j=1}^{r}$ is a set of matrices that is linear independent. Linear independent is the same as related vectors $\left\{\left|a_{1}\right\rangle, \ldots,\left|a_{\mathrm{N}}\right\rangle\right\} \subset C^{r}$ that constitute full set of vectors if $\left\langle a_{n}|M| a_{n}\right\rangle=0,1 \leq$ $n \leq N$ imply $M=0$ where $M \in C^{r \times r}$. The linear independence of the diagonal matrices $\left\{K_{i}^{\dagger} K_{j}\right\}_{i, j=1}^{r}$ means $r^{2} \leq N$ where $N$ represents the dimension of diagonal matrices and $r$ denotes the number of operators in Kraus representation or dimensionality of the vectors $\left|a_{n}\right\rangle$. It is defined that a phase damping channel will be extremal if $\left\{\left|\psi_{n}\right\rangle\left\langle\psi_{n}\right|\right\}$ is operator basis.

Helm et al. suggested a way to construct non-RU channels by using the Bloch vectors. It is possible to assign a corresponding generalized real Bloch vectors $\boldsymbol{b}_{n} \in \mathbb{R}^{d^{2}-1}$ to a given normalized complex vector $\left|\psi_{n}\right\rangle \in c^{d}$, in which $d$-dimensional quantum systems and $N=d^{2}[7,8]$. Since in two qubits systems the Pauli matrices satisfy the properties of a matrix basis, it means that they are traceless, i.e. $\operatorname{Tr} \sigma_{i}=0$ and orthogonal, they can be used for the Bloch vectors decomposition of qubits. The Bloch vector expansion of the environment density matrix is defined 
as [16-18]:

$$
\left|\psi_{n}\right\rangle\left\langle\psi_{n}\right|:=\boldsymbol{B}_{n} \cdot \boldsymbol{\sigma}, \quad \boldsymbol{B}_{n}=\frac{1}{2}\left(1, \boldsymbol{b}_{n}\right) \in \mathbb{R}^{d^{2}},
$$

in which the Bloch vectors form an operator basis and $\left\{\boldsymbol{B}_{n}\right\}$ denotes a linear independent set spanning $\mathbb{R}^{d^{2}}$ if a set of $d^{2}$ projectors $\left\{\left|\psi_{n}\right\rangle\left\langle\psi_{n}\right|\right\},\left(n=1, \ldots, d^{2}\right)$ form an operator basis. Extremality condition can be defined in terms of the Bloch vectors. In summary channel defined by dynamical vectors $\left\{\left|\psi_{1}\right\rangle, \ldots,\left|\psi_{4}\right\rangle\right\}$ is an extremal channel if the following equivalence is satisfied:

$$
V_{\mathrm{t}}:=\frac{1}{6} \operatorname{det}\left(\begin{array}{cccc}
1 & 1 & 1 & 1 \\
b_{1 x} & b_{2 x} & b_{3 x} & b_{4 x} \\
b_{1 y} & b_{2 y} & b_{3 y} & b_{4 y} \\
b_{1 z} & b_{2 z} & b_{3 z} & b_{4 z}
\end{array}\right) \neq 0 .
$$

In addition, geometrical interpretation could be defined that phase damping channels are extremal if the volume of the three-dimensional tetrahedron spanned by the Bloch vectors in $\mathbb{R}^{3}$ is not zero, or the same hyperplane in $\mathbb{R}^{3}$ is not pointed by the Bloch vectors $[7,8]$.

\subsection{Construction of non- $R U$ dynamics in phase damping channels}

Phase damping channels can be considered as the only diagonal doubly stochastic quantum channels and, there are nonunitary extremal doubly stochastic completely positive maps in $N>3$. In this section a new method is suggested to generate non-RU phase damping channels in two qubits system. The system includes bipartite system of qubits $(A$ and $B)$ which interacts with quantum environment that consisted of single qubit. Therefore, in this system $r^{2}=d^{2}=N$ can be satisfied. The total Hamiltonian $H$ of this system is considered as follows:

$$
H=H_{\mathrm{s}}+H_{\mathrm{I}}+H_{\mathrm{E}},
$$

in which $H_{\mathrm{s}}=\Omega_{1} \sigma_{z}^{(A)}+\Omega_{2} \sigma_{z}^{(B)}$ denotes the system Hamiltonian and $H_{\mathrm{I}}=\kappa_{1} \sigma_{Z}^{(A)} \sigma_{Z}^{(E)}+\kappa_{2} \sigma_{Z}^{(B)} \sigma_{Z}^{(E)}$ is interaction Hamiltonian that describes local coupling of the system and the environment. $H_{\mathrm{E}}=\boldsymbol{\Gamma} \cdot \boldsymbol{\sigma}^{\mathrm{E}}$ with $\boldsymbol{\Gamma}=\left(\Gamma_{X}, \Gamma_{Y}, \Gamma_{Z}\right), \boldsymbol{\sigma}^{\mathrm{E}}=\left(\sigma_{X} \sigma_{Y} \sigma_{Z}\right)$ represents the environment Hamiltonian.

It is assumed that the initial state is defined by a product state $\rho \otimes \sigma$ in which $\rho$ is the initial density matrix of the system and $\sigma$ is the initial density matrix of environment. The initial state is supposed to be a product state $|\psi\rangle=\left|\psi_{\mathrm{s}}\right\rangle \otimes\left|\psi_{\mathrm{E}}\right\rangle$ where $\left|\psi_{\mathrm{s}}\right\rangle$ describes density matrix of the system that includes two qubits so it can be described as follows:

$$
\begin{aligned}
& \left|\psi_{\mathrm{s}}\right\rangle=\alpha(|00\rangle+|11\rangle)+\beta(|10\rangle+|01\rangle), \\
& 2|\alpha|^{2}+2|\beta|^{2}=1,
\end{aligned}
$$

in which $\alpha$ and $\beta$ are coefficients. In order to satisfy the normalization condition, the sum of the squares of coefficients should be one. Furthermore, $\left|\psi_{\mathrm{E}}\right\rangle$ is state of the environment which is defined as follows:

$$
\left|\psi_{\mathrm{E}}\right\rangle=\left(\begin{array}{l}
1 \\
0
\end{array}\right) \text {. }
$$

To investigate the extremal channels, total state is ex- panded based on Hamiltonian's eigenvectors. Time evolution for total wave function $\left(\left|\psi_{\mathrm{s}}\right\rangle \otimes\left|\psi_{\mathrm{E}}\right\rangle\right)$ can be found readily by application of the following equation:

$$
|\psi(x, t)\rangle=\sum_{\mathrm{E}} C_{\mathrm{E}} u_{\mathrm{E}} \mathrm{e}^{-\mathrm{i} E t} / \hbar,
$$

in which $\hbar=1$ is supposed and $C_{\mathrm{E}}$ is coefficient. $u_{\mathrm{E}}$ and $E$ denote Hamiltonian eigenstates and corresponding eigenvalues, respectively. $u_{\mathrm{E}}$ and $E$ are extracted by means of $H u_{\mathrm{E}}(x)=E u_{\mathrm{E}}(x)$. In addition, $C_{\mathrm{E}}$ is obtained by using $\psi(x)=\sum_{\mathrm{E}} C_{\mathrm{E}} u_{\mathrm{E}}(x)$. Total density matrix can be obtained by using time evolution density matrix. As a result, environmental Bloch vectors can be calculated and the non-RU channels are examined by environmental Bloch vectors. Finally, total density matrix can be defined as follows:

$$
\rho=|\psi(x, t)\rangle\langle\psi(x, t)| .
$$

Finally, the evolution reduced density environment matrices $\rho_{\mathrm{E}}=\left|\psi_{n}(t)\right\rangle\left\langle\psi_{n}(t)\right|$ can be obtained by tracing over the system degrees of freedom, in which $\left|\psi_{n}(t)\right\rangle$ is time evolution of environmental state. Extremality can be investigated by means of the three-dimensional tetrahedron $\left(V_{\mathrm{t}}\right)$ volume spanned by the Bloch vectors in $\mathbb{R}^{3}$. $V_{\mathrm{t}}$ was obtained using Eq. (3.2) in the framework of time-alpha-dependent, as in Fig. 1.

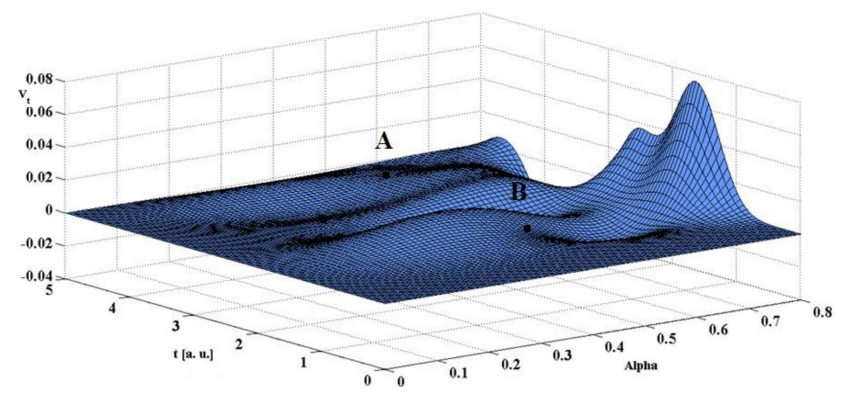

Fig. 1. Volume of three-dimensional tetrahedron spanned by the Bloch vectors in the three-dimensional space against $t$ and $\alpha$.

As it can be seen in the figure, there are some points that satisfy extremality condition i.e. $V_{\mathrm{t}}=0$ and in some points the extremality condition is not satisfied i.e. $V_{\mathrm{t}} \neq 0$. For instance, at point $A(\alpha=0.56, t=4.7)$ $V_{\mathrm{t}}$ is equal to zero and this channel belongs to $\mathrm{RU}$ dynamic. Therefore, correcting decoherence by obtaining classical information through measuring the quantum environment is possible in the mentioned point. On the contrary, at point $B(\alpha=0.5, t=1.5), V_{\mathrm{t}}$ is not equal to zero, therefore extremality condition is not satisfied and channel does not belong to RU maps.

Another geometrical interpretation for description of non-RU channels is defined by spanned corresponding Bloch vectors in $\mathbb{R}^{3}$. As it can be seen in Fig. 2, the Bloch vectors were obtained for two desired points.

As in Fig. 2, the corresponding Bloch vectors for points $A$ and $B$ are shown. In Fig. 2a the channel belongs to RU dynamics, because the vectors point to the same hy- 
(a)

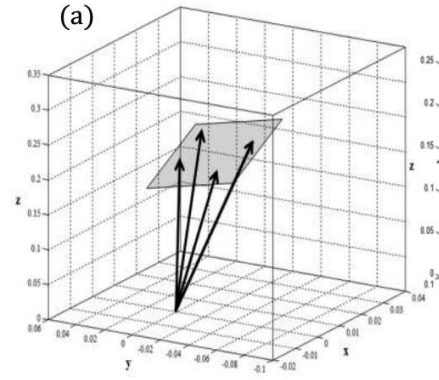

(b)

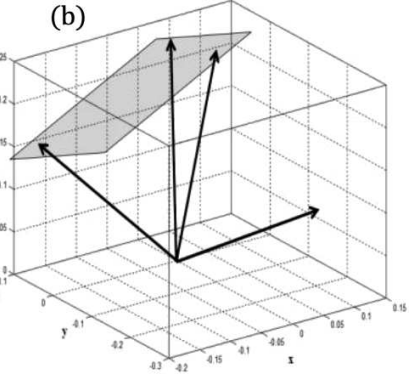

Fig. 2. Exemplary Bloch vectors in the three-dimensional space at point $A(\mathrm{a})$ and point $B(\mathrm{~b})$.

perplane in $\mathbb{R}^{3}$. Since Bloch vectors do not point to the same hyperplane in $\mathbb{R}^{3}$, the phase damping channel is extremal, as in Fig. 2b.

The Bell state is used to show the validity of RU dynamics determination based on Hamiltonian's eigenvectors. It is shown that the Bell state belongs to RU maps. The framework of time-dependent (Fig. 3) and alpha-dependent (Fig. 4) are shown for further investigation.

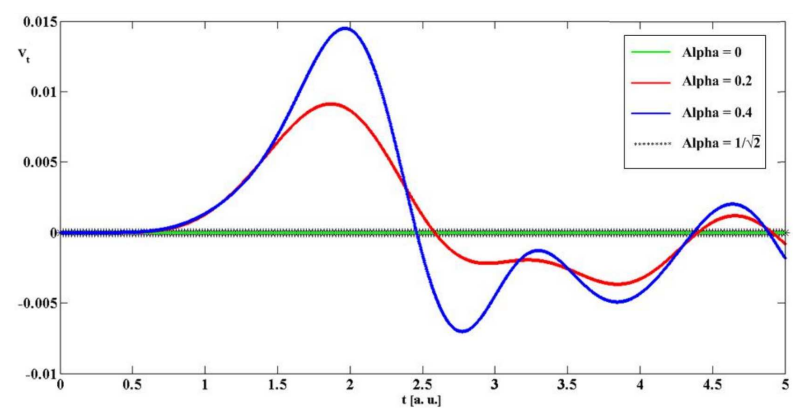

Fig. 3. Volume of three-dimensional tetrahedron spanned by the Bloch vectors in the three-dimensional space against $t$.

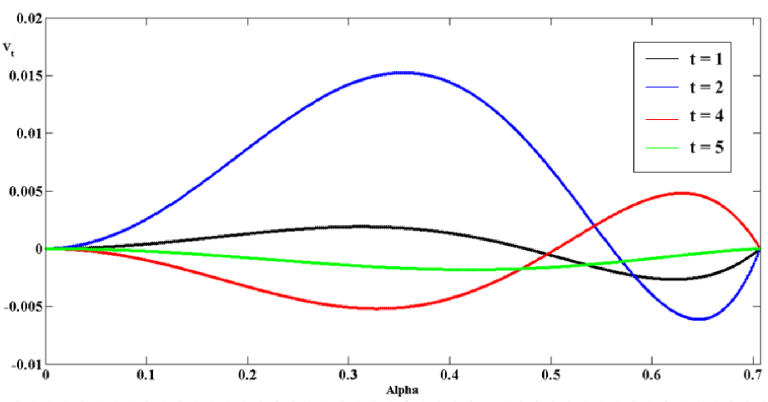

Fig. 4. Volume of three-dimensional tetrahedron spanned by the Bloch vectors in the three-dimensional space against $\alpha$.

According to Figs. 3 and 4 , at the points of $\alpha=0$ and $\alpha=\frac{1}{\sqrt{2}}$, phase damping channel belongs to RU channels at all-time i.e. $V_{\mathrm{t}}=0$. Based on classical information from measurement of the environment, error correction can be done. It is obvious that at these points $(\alpha=0$, $\left.\alpha=\frac{1}{\sqrt{2}}\right)$, Eq. (3.4) becomes a Bell state. Hence, these figures prove that the Bell state can be corrected by obtained classical information from measurement of the environment. In contrast, it is found out that, in the rest of the points of $\left(0<\alpha<\frac{1}{\sqrt{2}}\right)$, at most of the time, phase damping channel does not belong to RU dynamics i.e. $V_{\mathrm{t}} \neq 0$. Furthermore, the error correction method could not be established by obtaining classical information through measurement of the quantum environment.

\subsection{Extremality and rotation of environmental state}

The impact of the rotation of environmental state on environment-assisted error correction was examined. In this case, as previously mentioned, extremality could be investigated by utilizing $V_{\mathrm{t}}$ in the framework of time-alpha-dependent and exemplary Bloch vectors in three-dimension space, as in Figs. 5 and 6.

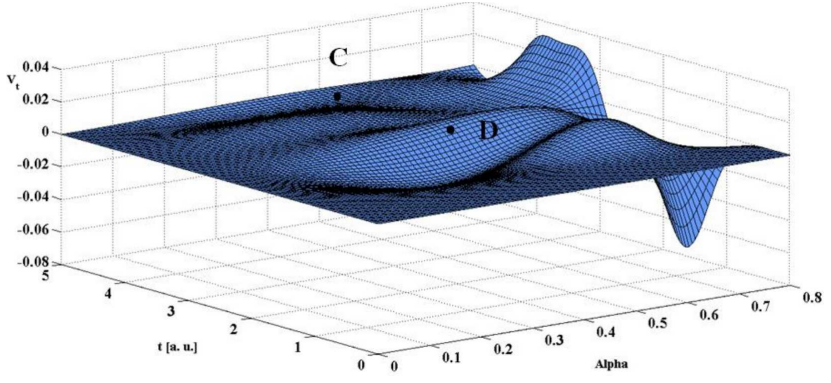

Fig. 5. Volume of three-dimensional tetrahedron spanned by the rotated Bloch vectors in the three-dimensional space against time $t$ and $\alpha$.
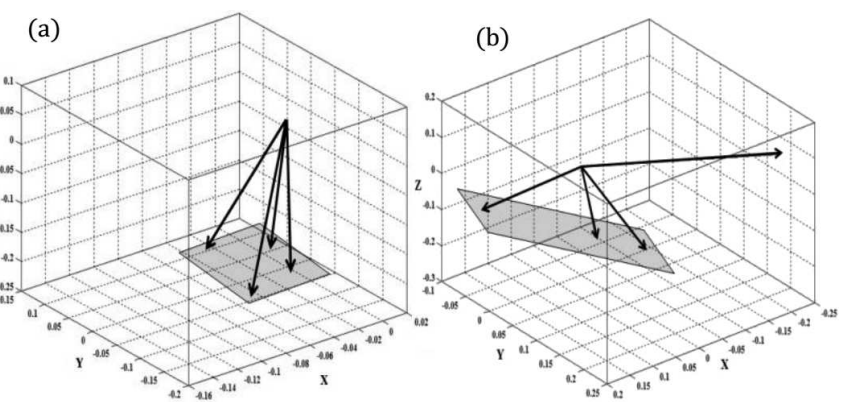

Fig. 6. Exemplary rotated Bloch vectors in the three-dimensional space at point $C(\mathrm{a})$ and point $D(\mathrm{~b})$.

As shown in the figure, there are some points that satisfy extremality condition i.e. $V_{\mathrm{t}}=0$ (at point $C$ $(\alpha=0.5, t=4.7))$ and at some points the extremality condition is not satisfied i.e. $V_{\mathrm{t}} \neq 0$ (at point $D(\alpha=0.43$, $t=2.55))$. The Bloch vectors were obtained for two desired points as another geometrical interpretation for the description of non-RU channels in Fig. 6. Obviously, the rotation of environmental state does not directly affect 
extremality and therefore it can be expressed as previously mentioned. For further investigation, the frameworks of time-dependent (Fig. 7) and alpha-dependent (Fig. 8) were depicted.

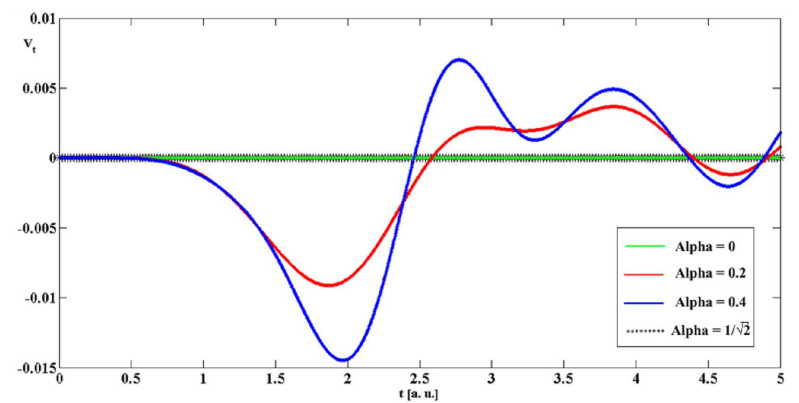

Fig. 7. Volume of three-dimensional tetrahedron spanned by the rotated Bloch vectors in the three-dimensional space against $t$.

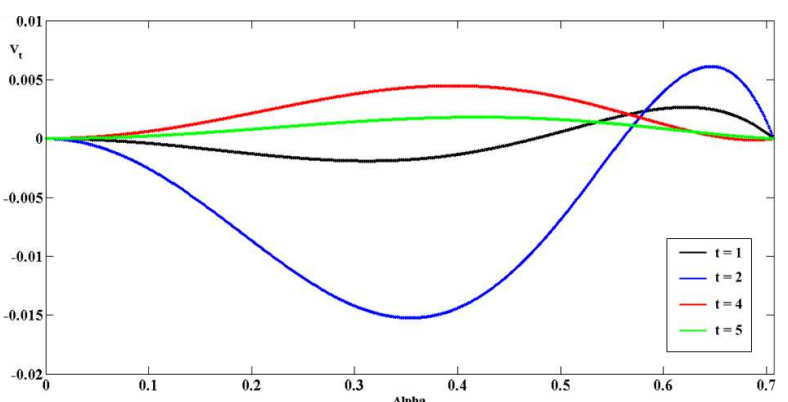

Fig. 8. Volume of three-dimensional tetrahedron spanned by the rotated Bloch vectors in the three-dimensional space against $\alpha$.

According to Figs. 7 and 8, phase damping channel can always be described by RU dynamics at the points of $\alpha=0$ and $\alpha=\frac{1}{\sqrt{2}}$. As mentioned earlier, the assumption state turns to the Bell state at these points. Therefore, this state can be corrected through environmental measurement. However, between the points of $0<\alpha<\frac{1}{\sqrt{2}}$, phase damping channel does not belong to RU maps. Therefore the rotation of the environmental state does not have effect on the results between the points of $0 \leq \alpha \leq \frac{1}{\sqrt{2}}$.

To investigate the non-RU channel, the following relations were established between parameters: (1) $0 \neq \kappa_{1} \neq$ $\kappa_{2} \neq 0 ;(2) \Gamma_{x} \neq 0$ or $\Gamma_{y} \neq 0 ;(3) \Gamma_{z} \neq 0$, otherwise, extremality condition is not satisfied.

\section{Conclusion}

Although environment-assisted error correction scheme is capable to correct the errors, there are some extremal channels that cannot be improved by means of classical information extracted from an environmental measurement. In this case, a phase damping channel consisting of two-qubit system interacting with single qubit environment is investigated. In this paper, the extremal phase damping channels were constructed by means of environmental Bloch vectors and the expansion of desired wave function based on Hamiltonian's eigenstates. The results demonstrated that the Bell state always belongs to RU dynamics and the quantum error method rests on extracting classical information from environment.

\section{References}

[1] A. Pernice, J. Helm, W.T. Strunz, J. Phys. B, At. Mol. Opt. 45, 154005 (2012).

[2] D. Mc Mahon, Quantum Computing Explained, Wiley, New Jersey 2007.

[3] H. Breuer, F. Petruccione, The Theory of Open Quantum Systems, Oxford University Press, New York 2002.

[4] J. Helm, W.T. Strunz, Phys. Rev. A. 81, 042314 (2010).

[5] B. Trendelkamp-Schroer, J. Helm, W.T. Strunz, Phys. Rev. A. 84, 062314 (2011).

[6] M. Gregoratti, R.F. Werner, J. Mod. Opt. 50, 915 (2003).

[7] J. Helm, W.T. Strunz, Phys. Rev. A. 80, 042108 (2009).

[8] J. Helm, W.T. Strunz, S. Rietzler, L.E. Wurflinger, Phys. Rev. A 83, 042103 (2011).

[9] F. Buscemi, G. Chiribella, G.M. D'Ariano, Phys. Rev. Lett. 95, 090501 (2005).

[10] L.J. Landau, R.F. Streater, Linear Algebr. Appl. 193, 107 (1993).

[11] W.K. Wootters, Quantum Inf. Comput. 1, 1 (2001).

[12] K.M.R. Auderaert, S. Scheel, New J. Phys. 10, 023011 (2008).

[13] F. Buscemi, Phys. Lett. A 360, 256 (2006).

[14] B. Rosgen, J. Math. Phys. 49, 102107 (2008).

[15] C.B. Mendi, M.M. Wolf, Commun. Math. Phys. 289 1057, (2009).

[16] R.A. Bertlmann, P. Krammer, J. Phys. A, Math. Theor. 41, 235303 (2008).

[17] G. Kimura, Phys. Lett. A 314, 339 (2003).

[18] L. Jakobczyk, M. Siennicki, Phys. Lett. A 286, 383 (2001). 